QUARTERLY OF APPLIED MATHEMATICS

VOLUME LXVII, NUMBER 2

JUNE 2009, PAGES 379-399

S 0033-569X(09)01130-1

Article electronically published on March 26, 2009

\title{
ON EXISTENCE OF A CLASSICAL SOLUTION AND NONEXISTENCE OF A WEAK SOLUTION TO THE DIRICHLET PROBLEM FOR THE LAPLACIAN WITH DISCONTINUOUS BOUNDARY DATA
}

\author{
BY
}

\author{
P. A. KRUTITSKII
}

KIAM, Miusskaya Sq. 4, Moscow 125047, Russia

\begin{abstract}
The Dirichlet problem for the Laplacian in a planar multiply connected interior domain bounded by smooth closed curves is considered in the case when the boundary data is piecewise continuous; i.e., it may have jumps in certain points of the boundary. It is assumed that the solution to the problem may not be continuous at the same points. The well-posed formulation of the problem is given, theorems on existence and uniqueness of a classical solution are proved, and the integral representation for a classical solution is obtained. The problem is reduced to a uniquely solvable Fredholm integral equation of the second kind and of index zero. It is shown that a weak solution to the problem does not exist typically, though the classical solution exists.
\end{abstract}

1. Introduction. It is known that if the Dirichlet problem for the Laplacian is considered in a planar domain bounded by sufficiently smooth closed curves, and if the function specified in the boundary condition is smooth enough, then the existence of a classical solution follows from the existence of a weak solution. In the present paper we consider the Dirichlet problem for the Laplacian in a planar multiply connected interior domain bounded by closed curves on the assumption that the boundary data is piecewise continuous; i.e., it may have jumps in certain points of the boundary. We look for a solution to the problem which may be discontinuous at these points. We give the well-posed formulation of the problem. We prove that there exists a unique classical solution to this problem and obtain an integral representation for the classical solution in the form of a double layer potential with some additions. Moreover, we reduce the problem to a uniquely solvable Fredholm integral equation of the second kind and of index zero for the density of a potential.

In addition, we prove that a weak solution to this problem may not exist. This result follows from the fact that the square of the gradient of the classical solution, basically, is

Received February 14, 2008.

2000 Mathematics Subject Classification. Primary 35J05, 35J25.

Key words and phrases. Laplace equation, Dirichlet problem, discontinuous boundary data.

(C)2009 Brown University 
not integrable near the points of discontinuity of the boundary data, since singularities of the gradient are rather strong there. This result is very important for numerical analysis. It shows that finite elements and finite difference methods cannot be applied to the numerical treatment of the Dirichlet problem in question directly 14, 15, 16, 17, since all these methods imply the existence of a weak solution. To use difference methods for numerical analysis one has to localize all strong singularities first and next use difference methods in a domain excluding the neighbourhoods of the singularities.

The Dirichlet problem with more general singularities of the boundary data than in the present paper has been studied in [1] by the theoretic functional approach. Results obtained in [1] have been extended to general elliptic systems in the upper half-plane in 2]. The paper 1] contains a formal existence theorem and a theorem on the number of solutions to the homogeneous problem. Unlike the present paper, the paper [1] is applicable to simply connected domains. The approach used in [1] does not provide us with any integral representation for a solution and does not reduce the problem to an integral equation even in the case of a simply connected domain, unlike the approach used in the present paper. In addition, the analysis of existence (or nonexistence) of a weak solution has not been carried out in 1 .

The Dirichlet problem for the Laplacian with continuous boundary data, but in cracked domains, i.e., in multiply connected domains bounded by closed curves and open arcs (cracks), has been studied in $[3$. The Dirichlet problem in cracked domains for the Laplacian has been analyzed in [4, 5] in the case when boundary data may be discontinuous at the tips of the cracks, i.e. if the functions specified on opposite sides of each crack may not satisfy matching conditions at the tips of the cracks in general. The problem for harmonic functions in cracked domains with posing the Neumann condition on the closed curves and the Dirichlet condition on the cracks has been studied in $\underline{6}$.

2. Formulation of the problem. In Cartesian coordinates $x=\left(x_{1}, x_{2}\right) \in R^{2}$ we consider the multiply connected domain bounded by simple closed curves $\Gamma_{1}, \ldots, \Gamma_{N} \in$ $C^{2, \lambda}, \lambda \in(0,1]$, so that the curves do not have common points and the curve $\Gamma_{1}$ encloses all others. We put

$$
\Gamma=\bigcup_{n=1}^{N} \Gamma_{n} .
$$

The connected domain bounded by $\Gamma$ will be called $\mathcal{D}$. We assume that each curve $\Gamma_{n}$ is parametrized by the arc length $s$ :

$$
\Gamma_{n}=\left\{x: x=x(s)=\left(x_{1}(s), x_{2}(s)\right), \quad s \in\left[a_{n}, b_{n}\right]\right\}, \quad n=1, \ldots, N,
$$

so that $a_{1}<b_{1}<a_{2}<b_{2}<\ldots<a_{N}<b_{N}$, and the domain $\mathcal{D}$ is on the right when the parameter $s$ increases on $\Gamma_{n}$. Hence $\left(b_{n}-a_{n}\right)$ is a length of $\Gamma_{n}$. Note that points $x \in \Gamma$ and values of the parameter $s$ are in one-to-one correspondence except for $a_{n}$ and $b_{n}$, which correspond to the same point $x\left(a_{n}\right)=x\left(b_{n}\right)$ for $n=1, \ldots, N$. We assume that $M(n) \geq 0$ is a given integer number for each $n=1, \ldots, N$. If $M(n)>0$, then we fix $M(n)$ points $p_{1}^{n}, \ldots, p_{M(n)}^{n}$ on the interval $\left(a_{n}, b_{n}\right)$ for $n=1, \ldots, N$ in such a way that

$$
a_{n}<p_{1}^{n}<\ldots<p_{M(n)}^{n}<b_{n} .
$$


The interval $\left[a_{n}, b_{n}\right]$ will be denoted by the same symbol as the corresponding curve, i.e. by $\Gamma_{n}$. In a similar manner, the set of the intervals on the $O s$ axis,

$$
\bigcup_{n=1}^{N}\left[a_{n}, b_{n}\right],
$$

will be denoted by the same symbol as the corresponding set of curves, that is, by $\Gamma$.

We put

$$
\begin{gathered}
C^{0}\left(\Gamma_{n}\right)=\left\{\mathcal{F}(s): \mathcal{F}(s) \in C^{0}\left[a_{n}, b_{n}\right], \quad \mathcal{F}\left(a_{n}\right)=\mathcal{F}\left(b_{n}\right)\right\}, \\
C^{0, \omega}\left(\Gamma_{n}\right)=\left\{\mathcal{F}(s): \mathcal{F}(s) \in C^{0, \omega}\left[a_{n}, b_{n}\right], \quad \mathcal{F}\left(a_{n}\right)=\mathcal{F}\left(b_{n}\right)\right\}, \quad \omega \in(0,1],
\end{gathered}
$$

and

$$
C^{0}(\Gamma)=\bigcap_{n=1}^{N} C^{0}\left(\Gamma_{n}\right), \quad C^{0, \omega}(\Gamma)=\bigcap_{n=1}^{N} C^{0, \omega}\left(\Gamma_{n}\right)
$$

By $\mathcal{D}_{n}$ we denote the interior domain bounded by the curve $\Gamma_{n}$, if $n=2, \ldots, N$. The exterior domain bounded by $\Gamma_{1}$ will be called $\mathcal{D}_{1}$.

The tangent vector to $\Gamma$ at the point $x(s)$ is denoted by $\tau_{x}=(\cos \alpha(s), \sin \alpha(s))$, where $\cos \alpha(s)=x_{1}^{\prime}(s)$ and $\sin \alpha(s)=x_{2}^{\prime}(s)$. Let $\mathbf{n}_{x}=(\sin \alpha(s),-\cos \alpha(s))$ be a normal vector to $\Gamma$ at $x(s)$. The direction of $\mathbf{n}_{x}$ is chosen such that it will coincide with the direction of $\tau_{x}$ if $\mathbf{n}_{x}$ is rotated anticlockwise through an angle of $\pi / 2$. Therefore $\mathbf{n}_{x}$ is the inward normal vector to $\mathcal{D}$ on $\Gamma$.

If integer $M(n)=0$, then we set $X_{n}=\emptyset$ and $\mathcal{X}_{n}=\emptyset$, while if integer $M(n)>0$, then we set $\mathcal{X}_{n}=\bigcup_{m=1}^{M(n)} p_{m}^{n}$ and $X_{n}=\bigcup_{m=1}^{M(n)} x\left(p_{m}^{n}\right)$. By $\mathcal{X}$ and $X$ we denote the pointsets consisting of all points included in $\mathcal{X}_{1}, \ldots, \mathcal{X}_{n}$ and in $X_{1}, \ldots, X_{N}$, respectively:

$$
\mathcal{X}=\bigcup_{n=1}^{N} \mathcal{X}_{n}, \quad X=\bigcup_{n=1}^{N} X_{n}
$$

We say that the function $u(x)$ belongs to the smoothness class $\mathbf{K}$ if

(1) $u \in C^{0}(\overline{\mathcal{D}} \backslash X) \cap C^{2}(\mathcal{D})$,

(2) in the neighbourhood of any point $x(d) \in X$ (i.e. $d \in \mathcal{X}$ ) the equality

$$
\lim _{r \rightarrow+0} \int_{\mathcal{D} \cap \partial S(d, r)} u(x) \frac{\partial u(x)}{\partial \mathbf{n}_{x}} d l=0
$$

holds, where the curvilinear integral of the first kind is taken over $\mathcal{D} \cap \partial S(d, r)$. We denote by $\partial S(d, r)$ a circumference of a radius $r$ with the center in the point $x(d)$; in addition, $\mathbf{n}_{x}$ is a normal vector to $\partial S(d, r)$ in the point $x \in \partial S(d, r)$. It is assumed that $\mathbf{n}_{x}$ is directed to the center of the circumference.

Let us formulate the Dirichlet problem for the Laplace equation in the domain $\mathcal{D}$.

Problem $\mathbf{U}$. Find a function $u(x)$ of class $\mathbf{K}$ so that $u(x)$ satisfies the Laplace equation

$$
u_{x_{1} x_{1}}(x)+u_{x_{2} x_{2}}(x)=0, \quad x \in \mathcal{D},
$$

and the boundary condition

$$
\left.u(x(s))\right|_{\Gamma}=F(s), \quad s \notin \mathcal{X} .
$$

All conditions of the problem $\mathbf{U}$ must be satisfied in the classical sense. Boundary condition (3) is not required in the points of the set $\mathcal{X}$. 
Using the energy equalities we can prove the following assertion.

Theorem 1. If $\Gamma \in C^{2, \lambda}, \lambda \in(0,1]$, then there is no more than one solution to the problem U.

By $\int_{\Gamma} \ldots d \sigma$ we mean

$$
\sum_{n=1}^{N} \int_{a_{n}}^{b_{n}} \ldots d \sigma
$$

Proof. It is sufficient to prove that the homogeneous problem $\mathbf{U}$ admits only the trivial solution. Let $u^{0}(x)$ be a solution to the homogeneous problem $\mathbf{U}$ with $F(s) \equiv 0$. Let $S(d, \epsilon)$ be a disc of a small enough radius $\epsilon$ with the center in the point $x(d) \in X$ (i.e. $d \in \mathcal{X})$. The union of such discs with the centers in all points of the pointset $X$ is denoted by $\mathcal{S}(X, \epsilon)$.

Set $\Gamma^{\epsilon}=\Gamma \backslash(\Gamma \cap \mathcal{S}(X, \epsilon)), \mathcal{D}^{\epsilon}=\mathcal{D} \backslash(\mathcal{D} \cap \mathcal{S}(X, \epsilon))$.

Since $\Gamma \in C^{2, \lambda}, u^{0}(x) \in C^{0}(\overline{\mathcal{D}} \backslash X) \quad$ (recall that $\left.u^{0}(x) \in \mathbf{K}\right)$, and since $\left.u^{0}\right|_{\Gamma \backslash X}=0 \in$ $C^{2, \lambda}(\Gamma \backslash X)$, and owing to the lemma on regularity of solutions of elliptic equations near the boundary [10, lemma 6.18], we obtain: $u^{0}(x) \in C^{1}(\overline{\mathcal{D}} \backslash X)$. Hence $u^{0}(x) \in C^{1}\left(\overline{\mathcal{D}^{\epsilon}}\right)$ for any small enough $\epsilon>0$. Since the boundary of the domain $\mathcal{D}^{\epsilon}$ is piecewise smooth, we write out Green's formula [9, p. 328] for the function $u^{0}(x)$ :

$$
\left\|\nabla u^{0}\right\|_{L_{2}\left(\mathcal{D}^{\epsilon}\right)}^{2}=-\int_{\Gamma^{\epsilon}} u^{0} \frac{\partial u^{0}}{\partial \mathbf{n}_{x}} d s+\int_{\mathcal{D} \cap \partial \mathcal{S}(X, \epsilon)} u^{0} \frac{\partial u^{0}}{\partial \mathbf{n}_{x}} d l .
$$

By $\mathbf{n}_{x}$ on $\partial \mathcal{S}(X, \epsilon)$ we denote the normal vector at the point $x \in \partial \mathcal{S}(X, \epsilon)$. The normal vector $\mathbf{n}_{x}$ is inward with respect to $\mathcal{S}(X, \epsilon)$. Since $u^{0}(x)$ satisfies the homogeneous boundary condition (3) on $\Gamma$, we observe that $\left.u^{0}\right|_{\Gamma \backslash X}=0$ and so $\left.u^{0}\right|_{\Gamma^{\epsilon}}=0$ for any small enough $\epsilon>0$. Therefore identity (4) takes the form

$$
\left\|\nabla u^{0}\right\|_{L_{2}\left(\mathcal{D}^{\epsilon}\right)}^{2}=\int_{\mathcal{D} \cap \partial \mathcal{S}(X, \epsilon)} u^{0} \frac{\partial u^{0}}{\partial \mathbf{n}_{x}} d l .
$$

Setting $\epsilon \rightarrow+0$, taking into account that $u^{0}(x) \in \mathbf{K}$ and using the relationship (1), we obtain

$$
\left\|\nabla u^{0}\right\|_{L_{2}(\mathcal{D})}^{2}=\lim _{\epsilon \rightarrow+0}\left\|\nabla u^{0}\right\|_{L_{2}\left(\mathcal{D}^{\epsilon}\right)}^{2}=0 .
$$

Hence $u^{0}(x) \equiv$ const in $\overline{\mathcal{D}} \backslash X$. From the homogeneous boundary conditions (3) we conclude that $u^{0}(x) \equiv 0$ in $\overline{\mathcal{D}} \backslash X$. The theorem is proved.

REMARK. The maximum principle cannot be used for the proof of the theorem, since the solution to the problem may not satisfy the boundary condition (3) in the points of the pointset $X$, and it may not be continuous at these points.

3. Existence of a classical solution. We say that the function $\mathcal{F}(s)$ belongs to the Banach space $B$ if for each $n=1, \ldots, N$ :

$$
\mathcal{F}(s) \in\left\{\begin{array}{ccc}
C^{0}\left[a_{n}, b_{n}\right], & \text { in case } & M(n)=0, \\
C^{0}\left[a_{n}, p_{1}^{n}-0\right] \cap C^{0}\left[p_{1}^{n}+0, b_{n}\right], & \text { in case } & M(n)=1, \\
C^{0}\left[a_{n}, p_{1}^{n}-0\right] \cap C^{0}\left[p_{1}^{n}+0, p_{2}^{n}-0\right] \cap \ldots & \\
\ldots \cap C^{0}\left[p_{M(n)}^{n}+0, b_{n}\right], & \text { in case } & M(n) \geq 2 .
\end{array}\right.
$$


The space $B$ can be represented as an intersection of spaces of continuous functions on a finite number of segments. The norm in the space $B$ is defined as a maximum of norms of the spaces of continuous functions on the segments.

We say that the function $\mathcal{F}(s)$ belongs to the class $B_{0}$ if $\mathcal{F}(s) \in B$ and

$$
\mathcal{F}\left(a_{n}\right)=\mathcal{F}\left(b_{n}\right), \quad n=1, \ldots, N .
$$

So, $B_{0} \subset B$. The functions of class $B_{0}$ may have jumps in each point of the pointset $\mathcal{X}$. In other words, these functions have limits in each point of $\mathcal{X}$ from the left and from the right, but the limits may be different. If $d \in \mathcal{X}$, then values of these functions in $d-0$ and in $d+0$ are understood in the sense of limits from the left and from the right, respectively.

Below we assume that the function $F(s)$ from the boundary condition (3) satisfies the following additional conditions:

$$
\begin{gathered}
F(s) \in B_{0}, \\
F(s) \in C^{1, \lambda}[d-\epsilon, d-0] \cap C^{1, \lambda}[d+0, d+\epsilon], \quad \lambda \in(0,1],
\end{gathered}
$$

for any $d \in \mathcal{X}$ and for some $\epsilon>0$ (small enough).

Note that the Hölder exponent $\lambda$ in the description of smoothness of the function $F(s)$ and in the description of smoothness of the boundary $\Gamma$ is the same. If the exponents are different in practice, then by $\lambda$ we denote the least.

We shall construct the solution to the problem $\mathbf{U}$ from the smoothness class $\mathbf{K}$ with the help of potential theory for harmonic functions. We look for a solution to the problem in the following form:

$$
u[\mu](x)=-\frac{1}{2 \pi} \int_{\Gamma} \mu(\sigma) \frac{\partial}{\partial \mathbf{n}_{y}} \ln |x-y(\sigma)| d \sigma+h[\mu](x),
$$

where the first term is the double layer potential on $\Gamma$. By $h[\mu](x)$ we denote the sum of point sources placed at the fixed points $Y_{n}$ lying inside $\Gamma_{n}(n=2, \ldots, N)$ :

$$
h[\mu](x)=-\frac{1}{2 \pi} \sum_{n=2}^{N} \int_{\Gamma_{n}} \mu(\sigma) d \sigma \ln \left|x-Y_{n}\right|, \quad Y_{n} \in \mathcal{D}_{n}, \quad n=2, \ldots, N .
$$

If $N=1$, then the sum is absent. We will look for the density $\mu(s)$ in the class $B_{0}$. The function $h[\mu](x)$ for such a density $\mu(s)$ obeys the Laplace equation in $R^{2} \backslash \bigcup_{n=2}^{N} Y_{n}$ and belongs to

$$
C^{\infty}\left(R^{2} \backslash \bigcup_{n=2}^{N} Y_{n}\right)
$$

furthermore, if $x(s) \in \Gamma$, then $h[\mu](x(s)) \in C^{2, \lambda}(\Gamma)$ in $s$. We need the function $h[\mu](x)$ to construct the uniquely solvable integral equation for finding the density $\mu(s)$.

It follows from [9] that if $\mu(s) \in B_{0}$, then the function (6) belongs to $C^{0}(\overline{\mathcal{D}} \backslash X) \cap C^{2}(\mathcal{D})$ and satisfies the Laplace equation (2) in $\mathcal{D}$. 
To satisfy the boundary condition (3), we put (6) in (3), use the limit formula for the double layer potential $[9$ and arrive at the integral equation for the density $\mu(s)$ :

$$
\frac{1}{2} \mu(s)-\frac{1}{2 \pi} \int_{\Gamma} \mu(\sigma) \frac{\partial \ln |x(s)-y(\sigma)|}{\partial \mathbf{n}_{y}} d \sigma+h[\mu](x(s))=F(s), \quad s \in \Gamma \backslash \mathcal{X} .
$$

It is shown in [8, Chapter IV, Section 5.6] that

$$
\frac{\partial \ln |x(s)-y(\sigma)|}{\partial \mathbf{n}_{y}} \in C^{0}(\Gamma \times \Gamma)
$$

for $\Gamma \in C^{2}$ (in our case $\Gamma \in C^{2, \lambda}$ ). Equation (7) can be rewritten in the form

$$
\mu(s)+\int_{\Gamma} \mu(\sigma) A(s, \sigma) d \sigma=2 F(s), \quad s \in \Gamma \backslash \mathcal{X},
$$

where

$$
A(s, \sigma)=-\frac{1}{\pi} \delta(\Gamma, \sigma) \frac{\partial}{\partial \mathbf{n}_{y}} \ln |x(s)-y(\sigma)|-\frac{1}{\pi} \sum_{n=2}^{N} \delta\left(\Gamma_{n}, \sigma\right) \ln \left|x(s)-Y_{n}\right| .
$$

If $N=1$, then the sum is absent. By $\delta(\gamma, \sigma)$ we denote the characteristic function of the set $\gamma$ :

$$
\delta(\gamma, \sigma)= \begin{cases}0, & \text { if } \sigma \notin \gamma \\ 1, & \text { if } \sigma \in \gamma .\end{cases}
$$

Since $\Gamma \in C^{2, \lambda}$, the kernel $A(s, \sigma)$ is continuous for all $s, \sigma \in \Gamma$.

Clearly, $F\left(a_{n}\right)=F\left(b_{n}\right)$ for $n=1, \ldots, N$, since we assume that $F(s) \in B_{0}$. Note that $A\left(a_{n}, \sigma\right)=A\left(b_{n}, \sigma\right)$ for any $\sigma \in \Gamma$ and for $n=1, \ldots, N$. Hence, if $\mu(s)$ is a solution of equation (8) from the Banach space $B$, then, according to the equality (8), $\mu(s)$ automatically satisfies the matching conditions

$$
\mu\left(a_{n}\right)=\mu\left(b_{n}\right), \quad n=1, \ldots, N,
$$

and so $\mu(s) \in B_{0}$. In other words, any solution of equation (8) in the space $B$ belongs to the class $B_{0}$ automatically. In view of this reason, below we look for a solution of equation (8) in the space $B$.

Since $A(s, \sigma) \in C^{0}(\Gamma \times \Gamma)$, it is obvious that the operator

$$
\mathcal{A} \mu=\int_{\Gamma} \mu(\sigma) A(s, \sigma) d \sigma
$$

maps $B$ into $C^{0}(\Gamma)$; i.e., this operator transforms any function of space $B$ into a function of space $C^{0}(\Gamma)$. Therefore the solution $\mu(s) \in B$ of equation (8) satisfies the following property at any point $d \in \mathcal{X}$ :

$$
\mu(d+0)-\mu(d-0)=F(d+0)-F(d-0), \quad d \in \mathcal{X} .
$$

Consequently, if $F(s) \in C^{0}(\Gamma)$, then any solution $\mu(s)$ of equation (8) in the space $B$ belongs to $C^{0}(\Gamma)$. Moreover, it can be verified directly, using the Arzelà-Ascoli theorem, that the operator $\mathcal{A} \mu$ is a compact operator mapping the space $B$ into the space $C^{0}(\Gamma) \subset B$. It follows from [11, 12, 13, that (8) is a Fredholm integral equation of the second kind and with index zero in the space $B$. In other words, the Fredholm alternative is applicable to equation (8) in the space $B$. 
If the function $F(s) \equiv 0 \in B$, then $F(s) \equiv 0 \in C^{0}(\Gamma)$. Consider the homogeneous equation (8) with respect to the function $\mu^{0}(s) \in B$ :

$$
\mu^{0}(s)+\int_{\Gamma} \mu^{0}(\sigma) A(s, \sigma) d \sigma=0, \quad s \in \Gamma \backslash \mathcal{X} .
$$

Equation (11) can also be written in the form

$$
\begin{gathered}
\frac{1}{2} \mu^{0}(s)-\frac{1}{2 \pi} \int_{\Gamma} \mu^{0}(\sigma) \frac{\partial}{\partial \mathbf{n}_{y}} \ln |x(s)-y(\sigma)| d \sigma \\
+h\left[\mu^{0}\right](x(s))=0, \quad s \in \Gamma \backslash \mathcal{X} .
\end{gathered}
$$

Note that $F(s) \equiv 0 \in C^{0}(\Gamma)$ in the homogeneous equation (12). It was mentioned above that in this case any solution of equation (12) in the space $B$ automatically belongs to $C^{0}(\Gamma)$. So, we have to look for a solution of equation $(12)$ in $C^{0}(\Gamma)$. Therefore (12) must be true for all $s \in \Gamma$ :

$$
\begin{aligned}
\frac{1}{2} \mu^{0}(s) & -\frac{1}{2 \pi} \int_{\Gamma} \mu^{0}(\sigma) \frac{\partial}{\partial \mathbf{n}_{y}} \ln |x(s)-y(\sigma)| d \sigma \\
& +h\left[\mu^{0}\right](x(s))=0, \quad s \in \Gamma .
\end{aligned}
$$

The equation $\left(12^{\prime}\right)$ is studied in the space $C^{0}(\Gamma)$ in Appendix 2, and it is shown that this equation has only the trivial solution in the space $C^{0}(\Gamma)$ (see Theorem A2). Consequently, equation (12) has only the trivial solution in the space $B$ as well. Owing to the Fredholm alternative, which is applicable to the equation (8) in the space $B$, we observe that the nonhomogeneous equation (8) is uniquely solvable in the space $B$. In other words, equation (8) has a unique solution $\mu(s) \in B$ for any right-hand side $F(s) \in B_{0} \subset B$.

We have proved the following assertion.

Theorem 2. If $\Gamma \in C^{2, \lambda}, \lambda \in(0,1]$, then (8) is a Fredholm equation of the second kind and of index zero in the space $B$. Moreover, equation (8) has a unique solution $\mu(s) \in B$ for any $F(s) \in B_{0}$. This solution satisfies properties (9), (10) and belongs to $B_{0}$.

Our goal is to show that if $F(s)$ satisfies conditions (5) and if $\mu(s) \in B$ is a solution of equation (8), then the function (6) satisfies condition (1) for any $d \in \mathcal{X}$. Firstly we will study the behaviour of the solution of equation (8) in a neighbourhood of any point $d \in \mathcal{X}$.

Let $\mu(s)$ be a solution of equation (8) in the space $B$. Let $d$ be an arbitrary point of the set $\mathcal{X}$ and let $\epsilon$ be the number from (5b). Set

$$
\gamma(d, \epsilon)=\{x: x=x(s), \quad s \in[d-\epsilon, d+\epsilon]\} .
$$

It is clear that for any $\mu(s) \in B$ :

$$
\begin{gathered}
\int_{\Gamma} \mu(\sigma) A(s, \sigma) d \sigma+\frac{1}{\pi} \int_{\gamma(d, \epsilon)} \mu(\sigma) \frac{\partial \ln |x(s)-y(\sigma)|}{\partial \mathbf{n}_{y}} d \sigma \\
=\left(-\frac{1}{2 \pi} \int_{\Gamma \backslash \gamma(d, \epsilon)} \mu(\sigma) \frac{\partial \ln |x(s)-y(\sigma)|}{\partial \mathbf{n}_{y}} d \sigma+h[\mu](x(s))\right) \in C^{1, \lambda}[d-\epsilon / 2, d+\epsilon / 2],
\end{gathered}
$$


in the variable $s$, since the function in the brackets does not have a singularity at $s=\sigma$ if $s \in[d-\epsilon / 2, d+\epsilon / 2]$. It is shown in Appendix 1 that for any $\mu(s) \in L_{\infty}[d-\epsilon, d+\epsilon]$ the function

$$
\frac{1}{\pi} \int_{\gamma(d, \epsilon)} \mu(\sigma) \frac{\partial \ln |x(s)-y(\sigma)|}{\partial \mathbf{n}_{y}} d \sigma
$$

belongs to $C^{1, \lambda / 4}[d-\epsilon, d+\epsilon]$ in the variable $s$. Subtracting (13b) from (13a) we observe that if $\mu(s) \in B$, then

$$
\left(\int_{\Gamma} \mu(\sigma) A(s, \sigma) d \sigma\right) \in C^{1, \lambda / 4}[d-\epsilon / 2, d+\epsilon / 2]
$$

in the variable $s$.

Set $f_{0}(s)=F(d-0)-F(d+0)+\left(F^{\prime}(d-0)-F^{\prime}(d+0)\right)(s-d)$. Let us introduce a function $f_{1}(s)$ for $s \in[d-\epsilon / 2, d+\epsilon / 2]$ :

$$
f_{1}(s)=\left\{\begin{array}{cc}
F(s)+f_{0}(s), & d \leq s \leq d+\epsilon / 2 \\
F(s), & d-\epsilon / 2 \leq s \leq d
\end{array}\right.
$$

It can be easily verified that $f_{1}(s) \in C^{1}[d-\epsilon / 2, d+\epsilon / 2]$. Moreover, using properties of Hölder continuous functions [7] one can show that $f_{1}^{\prime}(s)=\frac{d f_{1}(s)}{d s} \in C^{0, \lambda}[d-\epsilon / 2, d+\epsilon / 2]$, so, finally

$$
f_{1}(s) \in C^{1, \lambda}[d-\epsilon / 2, d+\epsilon / 2] .
$$

Note that the function $F(s)$ on the segment $[d-\epsilon / 2, d+\epsilon / 2]$ can be decomposed into a sum of two functions $f_{1}(s)$ and $f_{2}(s)$ :

$$
F(s)=f_{1}(s)+f_{2}(s), \quad s \in[d-\epsilon / 2, d+\epsilon / 2],
$$

where the function $f_{2}(s)$ is specified for $s \in[d-\epsilon / 2, d+\epsilon / 2]$ in the following way:

$$
f_{2}(s)=\left\{\begin{array}{cl}
-f_{0}(s), & d \leq s \leq d+\epsilon / 2 \\
0, & d-\epsilon / 2 \leq s \leq d
\end{array}\right.
$$

Assuming that $\mu(s) \in B$ is a solution of equation (8) we rewrite identity (8) for $s \in[d-\epsilon / 2, d+\epsilon / 2]$ in the form

$$
\mu(s)=-\int_{\Gamma} \mu(\sigma) A(s, \sigma) d \sigma+2\left(f_{1}(s)+f_{2}(s)\right), \quad s \in[d-\epsilon / 2, d+\epsilon / 2],
$$

where the function $F(s)$ is decomposed into the sum of functions $f_{1}(s)$ and $f_{2}(s)$ as proposed above. It follows from identity (15) that the function $\mu(s)$ on the segment $s \in[d-\epsilon / 2, d+\epsilon / 2]$ can be represented in the following way:

$$
\begin{gathered}
\mu(s)=\mu_{1}(s), \quad s \in[d-\epsilon / 2, d], \\
\mu(s)=\mu_{1}(s)+\mu_{2}(s), \quad s \in[d, d+\epsilon / 2],
\end{gathered}
$$

where

$$
\mu_{1}(s)=-\int_{\Gamma} \mu(\sigma) A(s, \sigma) d \sigma+2 f_{1}(s), \quad s \in[d-\epsilon / 2, d+\epsilon / 2],
$$

and

that is

$$
\mu_{2}(s)=2 f_{2}(s), \quad s \in[d, d+\epsilon / 2],
$$

$$
\mu_{2}(s)=-2 f_{0}(s), \quad s \in[d, d+\epsilon / 2] .
$$


Hence,

$$
\mu_{2}(d)=\mu_{2}(d+0)=2(F(d+0)-F(d-0)) .
$$

It was shown in (14) that the integral in (16a) belongs to

$$
C^{1, \lambda / 4}[d-\epsilon / 2, d+\epsilon / 2]
$$

in the variable $s$. Since $f_{1}(s) \in C^{1, \lambda}[d-\epsilon / 2, d+\epsilon / 2]$, we observe that

$$
\mu_{1}(s) \in C^{1, \lambda / 4}[d-\epsilon / 2, d+\epsilon / 2] .
$$

The function $\mu_{2}(s)$ is linear in $s$ on $[d, d+\epsilon / 2]$.

Let $F(s)$ satisfy conditions (5). We will study the behavior of the potential (6) in the neighbourhood of the point $x(d)$ for any $d \in \mathcal{X}$. Set

$$
\gamma^{\prime}(d, \epsilon)=\{x: x=x(s), \quad s \in[d, d+\epsilon]\}
$$

for $d \in \mathcal{X}$. Assuming that $\mu(s) \in B$ is a solution of equation (8), we decompose the potential $u[\mu](x)$ from (6) into three parts:

$$
u[\mu](x)=u_{0}[\mu](x)+u_{1}\left[\mu_{1}\right](x)+u_{2}\left[\mu_{2}\right](x),
$$

where

$$
\begin{gathered}
u_{0}[\mu](x)=-\frac{1}{2 \pi} \int_{\Gamma \backslash \gamma(d, \epsilon / 2)} \mu(\sigma) \frac{\partial}{\partial \mathbf{n}_{y}} \ln |x-y(\sigma)| d \sigma+h[\mu](x), \\
u_{1}\left[\mu_{1}\right](x)=-\frac{1}{2 \pi} \int_{\gamma(d, \epsilon / 2)} \mu_{1}(\sigma) \frac{\partial}{\partial \mathbf{n}_{y}} \ln |x-y(\sigma)| d \sigma, \\
u_{2}\left[\mu_{2}\right](x)=-\frac{1}{2 \pi} \int_{\gamma^{\prime}(d, \epsilon / 2)} \mu_{2}(\sigma) \frac{\partial}{\partial \mathbf{n}_{y}} \ln |x-y(\sigma)| d \sigma .
\end{gathered}
$$

It is clear that both $u_{0}[\mu](x)$ and $\nabla u_{0}[\mu](x)$ are continuously extensible onto $\gamma(d, \epsilon / 3)$ from $\mathcal{D}$. It follows from [4, theorem 2(I,II.1)], 5 , theorem 2(I,II.1)] that both $u_{1}\left[\mu_{1}\right](x)$ and $\nabla u_{1}\left[\mu_{1}\right](x)$ are continuously extensible onto $\gamma(d, \epsilon / 3)$ from $\mathcal{D}$ owing to (17). Let $S(d, \epsilon / 3)$ be a disc with the center in the point $x(d)$ and of radius $\epsilon / 3>0$. It follows from [4, theorem 2(I,II.1)], [5, theorem 2(I,II.1)] that both $u_{2}\left[\mu_{2}\right](x)$ and $\nabla u_{2}\left[\mu_{2}\right](x)$ are continuous in $S(d, \epsilon / 3) \backslash \gamma^{\prime}(d, \epsilon / 3)$ and are continuously extensible from $S(d, \epsilon / 3)$ onto $\gamma^{\prime}(d, \epsilon / 3) \backslash x(d)$ from the left and from the right. In particular, these functions are continuously extensible from $\mathcal{D}$ onto $\gamma(d, \epsilon / 3) \backslash x(d)$.

Set

$$
\cos \psi(x, y)=\frac{x_{1}-y_{1}}{|x-y|}=-|x-y|_{y_{1}}^{\prime}, \quad \sin \psi(x, y)=\frac{x_{2}-y_{2}}{|x-y|}=-|x-y|_{y_{2}}^{\prime} .
$$

Then $\psi(x, y)$ is a polar angle of the coordinate system with the origin in the point $y$.

According to [5, theor. 2(I)] for any $x \in S(d, \epsilon / 3)$ and $x \notin \gamma^{\prime}(d, \epsilon / 3)$ the following asymptotic formula holds:

$$
u_{2}\left[\mu_{2}\right](x)=\frac{\mu_{2}(d)}{2 \pi} \psi(x, x(d))+\Omega(x) .
$$

Here by $\psi(x, x(d))$ we mean some fixed branch of this function, so that the branch varies continuously in $x$ in $S(d, \epsilon / 3) \backslash \gamma^{\prime}(d, \epsilon / 3)$. The function $\Omega(x)$ is continuous as $x \rightarrow x(d)$. Moreover, $\Omega(x)$ is continuous in $S(d, \epsilon / 3)$ outside $\gamma^{\prime}(d, \epsilon / 3)$ and is continuously extensible from the left and from the right to $\gamma^{\prime}(d, \epsilon / 3)$. It follows from the asymptotic formula 
presented above that for any $x \in S(d, \epsilon / 3)$ and $x \notin \gamma^{\prime}(d, \epsilon / 3)$ the following inequality holds:

$$
\left|u_{2}\left[\mu_{2}\right](x)\right| \leq c,
$$

where $c$ is some positive constant.

It follows from [4, theorem 2(II.2)], [5, theorem 2(II.2)] that for $x \in S(d, \epsilon / 3)$ and $x \notin \gamma^{\prime}(d, \epsilon / 3)$ the following asymptotic formulae hold:

$$
\begin{gathered}
\frac{\partial u_{2}\left[\mu_{2}\right](x)}{\partial x_{1}}=-\frac{1}{2 \pi} \frac{\mu_{2}(d)}{|x-x(d)|} \sin \psi(x, x(d))+\Omega_{1}(x), \\
\frac{\partial u_{2}\left[\mu_{2}\right](x)}{\partial x_{2}}=\frac{1}{2 \pi} \frac{\mu_{2}(d)}{|x-x(d)|} \cos \psi(x, x(d))+\Omega_{2}(x),
\end{gathered}
$$

where

$$
\left|\Omega_{j}(x)\right| \leq c_{1} \ln \frac{1}{|x-x(d)|}, \quad j=1,2,
$$

and $c_{1}$ is a constant. Functions $\Omega_{1}(x)$ and $\Omega_{2}(x)$ are continuous in

$$
S(d, \epsilon / 3) \backslash \gamma^{\prime}(d, \epsilon / 3)
$$

and are continuously extensible from $S(d, \epsilon / 3)$ onto $\gamma^{\prime}(d, \epsilon / 3) \backslash x(d)$ from the left and from the right. In particular, these functions are continuously extensible from $\mathcal{D}$ onto $\gamma(d, \epsilon / 3) \backslash x(d)$.

It is clear from (20) that

$$
\left.\frac{\partial u_{2}\left[\mu_{2}\right](x)}{\partial \mathbf{n}_{x}}\right|_{x \in \partial S(d, r)}=-\Omega_{1}(x) \cos \psi(x, x(d))-\Omega_{2}(x) \sin \psi(x, x(d)),
$$

where the normal vector $\mathbf{n}_{x}=(-\cos \psi(x, x(d)),-\sin \psi(x, x(d)))$ on $\partial S(d, r)$ is directed to $x(d)$ (the center of the circle $S(d, r)$ ).

Therefore, using (19), (22a) and (21) we obtain that if $r \rightarrow+0$, then

$$
\lim _{r \rightarrow+0}\left|\int_{\mathcal{D} \cap \partial S(d, r)} u_{2}\left[\mu_{2}\right](x) \frac{\partial u_{2}\left[\mu_{2}\right]}{\partial \mathbf{n}_{x}} d l\right| \leq 2 \pi c c_{1} \lim _{r \rightarrow+0} r \ln \frac{1}{r}=0 .
$$

Let $\mu(s) \in B$ be a solution of equation (8) for $F(s)$ satisfying conditions (5). Let us check that the potential $u[\mu](x)$ given by formula (6) satisfies condition (1) for any $d \in \mathcal{X}$.

For points $x \in \mathcal{D}$ lying in a neighbourhood of $x(d)$ we set

$$
U(x)=u_{0}[\mu](x)+u_{1}\left[\mu_{1}\right](x)=u[\mu](x)-u_{2}\left[\mu_{2}\right](x),
$$

where we used the notation from (18). It was shown above that both $U(x)$ and $\nabla U(x)$ are continuously extensible from $\mathcal{D}$ onto $\gamma(d, \epsilon / 3)$, whence

$$
|U(x)|,|\nabla U(x)| \leq \text { const }, \quad x \in \mathcal{D} \cap S(d, \epsilon / 3),
$$


where the inequality holds for some fixed const $>0$. Let $r$ be small enough $(r<\epsilon / 3)$; then substituting (6) into the integral in (1) and using (23) we obtain

$$
\begin{gathered}
\int_{\mathcal{D} \cap \partial S(d, r)} u[\mu](x) \frac{\partial u[\mu](x)}{\partial \mathbf{n}_{x}} d l \\
=\int_{\mathcal{D} \cap \partial S(d, r)} u_{2}\left[\mu_{2}\right](x) \frac{\partial u_{2}\left[\mu_{2}\right](x)}{\partial \mathbf{n}_{x}} d l+\int_{\mathcal{D} \cap \partial S(d, r)} u_{2}\left[\mu_{2}\right](x) \frac{\partial U(x)}{\partial \mathbf{n}_{x}} d l \\
+\int_{\mathcal{D} \cap \partial S(d, r)} U(x) \frac{\partial u_{2}\left[\mu_{2}\right](x)}{\partial \mathbf{n}_{x}} d l+\int_{\mathcal{D} \cap \partial S(d, r)} U(x) \frac{\partial U(x)}{\partial \mathbf{n}_{x}} d l .
\end{gathered}
$$

If $r \rightarrow+0$, then the first term tends to zero by (22b). Owing to (24) the condition (1) holds for the function $U(x)$, so the fourth term tends to zero as $r \rightarrow+0$. The second term tends to zero as $r \rightarrow+0$, since $u_{2}\left[\mu_{2}\right](x)$ is bounded in $S(d, r) \cap \mathcal{D}$ according to (19), and since $\nabla U(x)$ is also bounded in $S(d, r) \cap \mathcal{D}$ in view of (24). Noting that $U(x)$ satisfies inequality (24) and using (22a) and (21), we deduce that the third term tends to zero when $r \rightarrow+0$ as well:

$$
\lim _{r \rightarrow+0}\left|\int_{\mathcal{D} \cap \partial S(d, r)} U(x) \frac{u_{2}\left[\mu_{2}\right](x)}{\partial \mathbf{n}_{x}} d l\right| \leq 2 \pi c_{1} \cdot \text { const } \cdot \lim _{r \rightarrow+0} r \ln \frac{1}{r}=0 .
$$

Consequently, the equality (1) holds for the function $u[\mu](x)$ from (6), where $\mu(s) \in B$ is a solution of equation (8) for $F(s)$ satisfying conditions (5). This means that the function $u[\mu](x)$ from (6) satisfies all the conditions of the problem $\mathbf{U}$.

We have proved

Theorem 3. Let $\Gamma \in C^{2, \lambda}, \quad \lambda \in(0,1]$, and let the function $F(s)$ satisfy conditions (5). Then a solution to the problem $\mathbf{U}$ exists and is given by the formula (6), where $\mu(s) \in B_{0}$ is a unique solution to the integral equation (8) ensured by Theorem 2 .

Uniqueness of a solution to the problem $\mathbf{U}$ follows from Theorem 1.

4. Nonexistence of a weak solution. In fact, the solution to the problem $\mathbf{U}$ presented in Theorem 3 is a classical solution. Let us discuss, under which conditions this solution to the problem $\mathbf{U}$ is not a weak solution. Let $u(x)$ be a solution to the problem U defined in Theorem 3. Consider a disc $S(d, \epsilon / 3)$ with the center at the point $x(d) \in X$ and of radius $\epsilon / 3>0 \quad(d \in \mathcal{X})$. In doing so, $\epsilon$ is taken from the smoothness condition (5b) for the function $F(s)$. Note that $\epsilon$ can be taken small enough. Consider the representation for $u(x)$ in $\mathcal{D} \cap S(d, \epsilon / 3)$ given by formula (6). Consider such a sector with the center in $x(d)$ and of radius $\epsilon / 3$ and of angle $\beta=\left(\beta_{2}-\beta_{1}\right)>0$,

$$
P(d, \epsilon / 3)=\left\{x: x \in S(d, \epsilon / 3), \quad \beta_{1}<\psi(x, x(d))<\beta_{2}\right\},
$$

which lies in the domain $\mathcal{D}: \quad P(d, \epsilon / 3) \subset \mathcal{D}$. Recall that $\psi(x, x(d))$ is a polar angle of the polar system of coordinates with the center in the point $x(d)$. So, $P(d, \epsilon / 3) \subset$ $(\mathcal{D} \cap S(d, \epsilon / 3))$. Existence of such a sector $P(d, \epsilon / 3)$ follows from [7, $\S 2 . I V]$. 
Assume that $u(x)$ is decomposed according to (18). Using formulae (20) and setting $r=|x-x(d)|, \quad \psi=\psi(x, x(d))$, we consider the integral over the sector $P(d, \epsilon / 3)$ :

$$
\begin{gathered}
\int_{P(d, \epsilon / 3)}\left|\nabla u_{2}\left[\mu_{2}\right](x)\right|^{2} d x=\int_{\beta_{1}}^{\beta_{2}} \int_{0}^{\epsilon / 3}\left\{\left(\frac{\mu_{2}(d)}{2 \pi r}\right)^{2}\right. \\
\left.+\frac{\mu_{2}(d)}{\pi r}\left(-\Omega_{1}(x) \sin \psi+\Omega_{2}(x) \cos \psi\right)+\Omega_{1}^{2}(x)+\Omega_{2}^{2}(x)\right\} r d r d \psi=I_{1}+I_{2}, \\
I_{1}=\frac{\beta_{2}-\beta_{1}}{(2 \pi)^{2}} \int_{0}^{\epsilon / 3} \frac{1}{r} \mu_{2}^{2}(d) d r \\
I_{2}=\int_{\beta_{1}}^{\beta_{2}} \int_{0}^{\epsilon / 3}\left\{\frac{\mu_{2}(d)}{\pi}\left(-\Omega_{1}(x) \sin \psi+\Omega_{2}(x) \cos \psi\right)+r\left(\Omega_{1}^{2}(x)+\Omega_{2}^{2}(x)\right)\right\} d r d \psi .
\end{gathered}
$$

The integral $I_{2}$ converges according to the estimates (21):

$$
\left|I_{2}\right| \leq 2\left(\beta_{2}-\beta_{1}\right) c_{1} \int_{0}^{\epsilon / 3} \ln \frac{1}{r}\left(\frac{1}{\pi}\left|\mu_{2}(d)\right|+c_{1} r \ln \frac{1}{r}\right) d r \leq \text { const. }
$$

Hence, if integral (25) converges, then the integral $I_{1}$ converges as well (as a difference of two convergent integrals), but the integral $I_{1}$ converges if and only if $\mu_{2}(d)=0$, while in the other cases, $I_{1}$ diverges. Thus, the integral (25) converges if and only if $\mu_{2}(d)=0$. Consequently $\left|\nabla u_{2}\left[\mu_{2}\right](x)\right|$ belongs to $L_{2}(P(d, \epsilon / 3))$ with small $\epsilon>0$ if and only if $\mu_{2}(d)=0$.

Consider representation (23) in the neighbourhood of the point $x(d)$. Since $U(x)$ and $\nabla U(x)$ are continuously extensible from $\mathcal{D}$ onto $\gamma(d, \epsilon / 3)$, we have $U \in L_{2}(P(d, \epsilon / 3))$ and $|\nabla U| \in L_{2}(P(d, \epsilon / 3))$. Let $x \in P(d, \epsilon / 3)$. It follows from $(23)$ that $\left|\nabla u_{2}\left[\mu_{2}\right](x)\right| \leq$ $|\nabla u(x)|+|\nabla U(x)|$, whence

$$
\begin{aligned}
\left|\nabla u_{2}\left[\mu_{2}\right](x)\right|^{2} \leq & |\nabla u(x)|^{2}+|\nabla U(x)|^{2}+2|\nabla u(x)| \cdot|\nabla U(x)| \\
& \leq 2\left(|\nabla u(x)|^{2}+|\nabla U(x)|^{2}\right),
\end{aligned}
$$

since $2|\nabla u(x)| \cdot|\nabla U(x)| \leq|\nabla u(x)|^{2}+|\nabla U(x)|^{2}$. Assume that $|\nabla u|$ belongs to $L_{2}(P(d, \epsilon / 3))$. Then integrating this inequality over $P(d, \epsilon / 3)$, we obtain $\left.\left\|\nabla u_{2}\left[\mu_{2}\right]\right\|^{2}\right|_{L_{2}(P(d, \epsilon / 3))} \leq 2\left(\left.\|\nabla u\|^{2}\right|_{L_{2}(P(d, \epsilon / 3))}+\left.\|\nabla U\|^{2}\right|_{L_{2}(P(d, \epsilon / 3))}\right)$. Consequently, if $|\nabla u| \in L_{2}(P(d, \epsilon / 3))$, then $\left|\nabla u_{2}\left[\mu_{2}\right]\right| \in L_{2}(P(d, \epsilon / 3))$.

However, it was shown that if $\mu_{2}(d) \neq 0$, then $\left|\nabla u_{2}\left[\mu_{2}\right]\right|$ does not belong to $L_{2}(P(d, \epsilon / 3))$. Therefore, if $\mu_{2}(d) \neq 0$, then our assumption that $|\nabla u| \in L_{2}(P(d, \epsilon / 3))$ does not hold, i.e. $|\nabla u| \notin L_{2}(P(d, \epsilon / 3))$. Thus, if among the numbers $d \in \mathcal{X}$ there exists such a number $d$ that $\mu_{2}(d)=2(F(d+0)-F(d-0)) \neq 0$ (here (16b) is used), then for some $\epsilon>0$ we have $|\nabla u| \notin L_{2}(P(d, \epsilon / 3))$, so $u \notin W_{2}^{1}(P(d, \epsilon / 3))$, where $W_{2}^{1}$ is the Sobolev space of functions from $L_{2}$, which have generalized derivatives from $L_{2}$. Obviously, if $u \notin W_{2}^{1}(P(d, \epsilon / 3))$ for some $\epsilon>0$, then $u \notin W_{2}^{1}(\mathcal{D})$. We have proved

ThEOREM 4. Let the conditions of Theorem 3 hold. If among the numbers $d \in \mathcal{X}$, there exists such a number $d$ that $F(d+0) \neq F(d-0)$, then the solution to the problem $\mathbf{U}$, ensured by Theorem 3 , does not belong to $W_{2}^{1}(\mathcal{D})$. 
If the conditions of Theorem 4 hold, then the unique solution to the problem $\mathbf{U}$, constructed in Theorem 3, does not belong to $W_{2}^{1}(\mathcal{D})$, and so it is not a weak solution. We arrive at

Corollary. Let the conditions of Theorem 4 hold. Then a weak solution to the problem $\mathbf{U}$ in the space $W_{2}^{1}(\mathcal{D})$ does not exist.

REMARK. Even if the number $d$, mentioned in Theorem 5, does not exist, then the solution $u(x)$ to the problem $\mathbf{U}$, ensured by Theorem 4 , may not be a weak solution to the problem $\mathbf{U}$. The Hadamard example of a nonexistence of a weak solution to a harmonic Dirichlet problem in a disc with continuous boundary data is given in [19, §12.5] (the classical solution exists in this example).

Appendix 1. Let us study the smoothness of the direct value of the double layer potential on the open arc. We will prove

TheOREm A1. Let $\gamma$ be an open arc of class $C^{2, \lambda}, \lambda \in(0,1]$. Assume that $\gamma$ is parametrized by the arc length $s$ :

$$
\gamma=\left\{x: x(s)=\left(x_{1}(s), x_{2}(s)\right), s \in[a, b]\right\}
$$

and assume that $\mu(s) \in L_{\infty}[a, b]$. Let

$$
\mathcal{I}_{1}(s)=-\frac{1}{2 \pi} \int_{\gamma} \mu(\sigma) \frac{\partial \ln |x(s)-y(\sigma)|}{\partial \mathbf{n}_{y}} d \sigma
$$

be the direct value of the double layer potential on $\gamma$. Then

$$
\mathcal{I}_{1}(s) \in C^{1, \lambda / 4}[a, b] .
$$

Proof. Let us prove that $\mathcal{I}_{1}(s) \in C^{1, \lambda / 4}[a, b]$. Taking into account that $\mathbf{n}_{y}=\left(y_{2}^{\prime}(\sigma),-y_{1}^{\prime}(\sigma)\right)$, we find

$$
\begin{gathered}
\frac{\partial \ln |x(s)-y(\sigma)|}{\partial \mathbf{n}_{y}}=\frac{T(s, \sigma)}{g(s, \sigma)}, \quad g(s, \sigma)=\frac{|x(s)-y(\sigma)|^{2}}{(s-\sigma)^{2}}, \\
T(s, \sigma)=\frac{\left[x_{2}(s)-y_{2}(\sigma)\right] y_{1}^{\prime}(\sigma)-\left[x_{1}(s)-y_{1}(\sigma)\right] y_{2}^{\prime}(\sigma)}{(s-\sigma)^{2}} .
\end{gathered}
$$

Note that $y(\sigma)$ is a point on $\Gamma$ corresponding to $s=\sigma$. So, we may put $x(\sigma)=y(\sigma)$. For $j=1,2$ we have [18, $\S 3]$

$$
x_{j}(s)-x_{j}(\sigma)=(s-\sigma) Z_{j}^{1}(s, \sigma)=-x_{j}^{\prime}(\sigma)(\sigma-s)+(\sigma-s)^{2} Z_{j}^{2}(\sigma, s),
$$

where

$$
\begin{aligned}
& Z_{j}^{1}(s, \sigma)=\int_{0}^{1} x_{j}^{\prime}(\sigma+\xi(s-\sigma)) d \xi \in C^{1, \lambda}([a, b] \times[a, b]), \\
& Z_{j}^{2}(\sigma, s)=\int_{0}^{1} \xi x_{j}^{\prime \prime}(s+\xi(\sigma-s)) d \xi \in C^{0, \lambda}([a, b] \times[a, b]) .
\end{aligned}
$$

Note that the function

$$
g(s, \sigma)=\frac{|x(s)-x(\sigma)|^{2}}{(s-\sigma)^{2}}=\left\{\left[Z_{1}^{1}(s, \sigma)\right]^{2}+\left[Z_{2}^{1}(s, \sigma)\right]^{2}\right\} \in C^{1, \lambda}([a, b] \times[a, b])
$$


does not equal zero anywhere on $\Gamma$ and $g(s, s)=1$. Therefore

$$
\frac{1}{g(s, \sigma)} \in C^{1}([a, b] \times[a, b]) .
$$

Further,

$$
\begin{aligned}
\frac{\partial}{\partial s} \frac{1}{g(s, \sigma)} & =\frac{\partial}{\partial s} \frac{(s-\sigma)^{2}}{|x(s)-x(\sigma)|^{2}}=-\frac{g_{s}^{\prime}(s, \sigma)}{g^{2}(s, \sigma)} \\
& =-2 \frac{Z_{1}^{1}(s, \sigma)\left[Z_{1}^{1}(s, \sigma)\right]_{s}^{\prime}+Z_{2}^{1}(s, \sigma)\left[Z_{2}^{1}(s, \sigma)\right]_{s}^{\prime}}{g^{2}(s, \sigma)} \in C^{0, \lambda}([a, b] \times[a, b]) .
\end{aligned}
$$

Consequently, $\frac{1}{g(s, \sigma)} \in C^{1, \lambda}([a, b] \times[a, b])$. Similarly,

$$
\begin{aligned}
T(s, \sigma) & =\frac{\left[x_{2}(s)-x_{2}(\sigma)\right] x_{1}^{\prime}(\sigma)-\left[x_{1}(s)-x_{1}(\sigma)\right] x_{2}^{\prime}(\sigma)}{(s-\sigma)^{2}} \\
& =\left[Z_{2}^{2}(\sigma, s) x_{1}^{\prime}(\sigma)-Z_{1}^{2}(\sigma, s) x_{2}^{\prime}(\sigma)\right] \in C^{0, \lambda}([a, b] \times[a, b]) .
\end{aligned}
$$

Consider $\frac{\partial T(s, \sigma)}{\partial s}=J_{1}(s, \sigma)-2 J_{2}(s, \sigma)$, where

$$
\begin{aligned}
& J_{1}(s, \sigma)=\frac{x_{2}^{\prime}(s) x_{1}^{\prime}(\sigma)-x_{1}^{\prime}(s) x_{2}^{\prime}(\sigma)}{(s-\sigma)^{2}} \\
&=\frac{\left[x_{2}^{\prime}(s)-x_{2}^{\prime}(\sigma)\right] x_{1}^{\prime}(\sigma)-\left[x_{1}^{\prime}(s)-x_{1}^{\prime}(\sigma)\right] x_{2}^{\prime}(\sigma)}{(s-\sigma)^{2}} \\
&=\frac{1}{s-\sigma}\left\{x_{1}^{\prime}(\sigma) \int_{0}^{1} x_{2}^{\prime \prime}[s+\xi(\sigma-s)] d \xi\right. \\
& J_{2}(s, \sigma)=\frac{\left[x_{2}(s)-x_{2}^{\prime}(\sigma) \int_{0}^{1} x_{1}^{\prime \prime}[s+\xi(\sigma-s)] d \xi\right\}}{(s-\sigma)^{3}}(\sigma)-\left[x_{1}(s)-x_{1}(\sigma)\right] x_{2}^{\prime}(\sigma) \\
&=\frac{1}{s-\sigma}\left\{x_{1}^{\prime}(\sigma) \int_{0}^{1} \xi x_{2}^{\prime \prime}[s+\xi(\sigma-s)] d \xi\right. \\
&\left.-x_{2}^{\prime}(\sigma) \int_{0}^{1} \xi x_{1}^{\prime \prime}[s+\xi(\sigma-s)] d \xi\right\} .
\end{aligned}
$$

Then

$$
\begin{aligned}
\frac{\partial T(s, \sigma)}{\partial s}=\frac{1}{s-\sigma}\{ & x_{1}^{\prime}(\sigma) \int_{0}^{1}(1-2 \xi) x_{2}^{\prime \prime}[s+\xi(\sigma-s)] d \xi \\
& \left.\quad x_{2}^{\prime}(\sigma) \int_{0}^{1}(1-2 \xi) x_{1}^{\prime \prime}[s+\xi(\sigma-s)] d \xi\right\}=\frac{K(s, \sigma)}{s-\sigma},
\end{aligned}
$$

where $K(s, \sigma) \in C^{0, \lambda}([a, b] \times[a, b])$ and $K(s, s)=0$. According to [7, $\left.\S 5.7\right]$, the following representation holds:

$$
\frac{\partial T(s, \sigma)}{\partial s}=\frac{K^{*}(s, \sigma)}{|s-\sigma|^{1-\lambda / 4}}
$$


$K^{*}(s, \sigma) \in C^{0,3 \lambda / 4}([a, b] \times[a, b])$. Using properties of Hölder functions [7, we obtain the representation

$$
\begin{aligned}
\frac{\partial}{\partial s} \frac{\partial \ln |x(s)-y(\sigma)|}{\partial \mathbf{n}_{y}} & =\frac{1}{g(s, \sigma)} \frac{\partial T(s, \sigma)}{\partial s}+T(s, \sigma) \frac{\partial}{\partial s} \frac{1}{g(s, \sigma)} \\
& =\frac{K_{1}(s, \sigma)}{|s-\sigma|^{1-\lambda / 4}}+K_{2}(s, \sigma),
\end{aligned}
$$

where $K_{1}(s, \sigma) \in C^{0,3 \lambda / 4}([a, b] \times[a, b]), \quad K_{2}(s, \sigma) \in C^{0, \lambda}([a, b] \times[a, b])$. By formal differentiation under the integral, we find

$$
\begin{aligned}
\frac{d \mathcal{I}_{1}(s)}{d s} & =-\frac{1}{2 \pi} \int_{\gamma} \mu(\sigma) \frac{\partial}{\partial s} \frac{\partial \ln |x(s)-y(\sigma)|}{\partial \mathbf{n}_{y}} d \sigma \\
& =-\frac{1}{2 \pi} \int_{\gamma} \mu(\sigma) \frac{K_{1}(s, \sigma)}{|s-\sigma|^{1-\lambda / 4}} d \sigma-\frac{1}{2 \pi} \int_{\gamma} \mu(\sigma) K_{2}(s, \sigma) d \sigma .
\end{aligned}
$$

The validity of differentiation under the integral can be proved in the same way as at the end of $\S 1.6$ in 9 (the Fubini theorem on change of integration order is used). Taking into account the obtained representation for $\frac{d \mathcal{I}_{1}(s)}{d s}$ and applying results of [7, $\left.\S 51.1\right]$, we obtain that $\frac{d \mathcal{I}_{1}(s)}{d s} \in C^{0, \lambda / 4}[a, b]$. Theorem A1 is proved.

Appendix 2. In this section we study equation $\left(12^{\prime}\right)$ under weakened conditions, namely, in assumption that $\Gamma \in C^{2,0}$. Here we prove the following assertion.

Theorem A2. If $\Gamma \in C^{2,0}$, then there is only the trivial solution of the homogeneous Fredholm equation $\left(12^{\prime}\right)$ in $C^{0}(\Gamma)$.

Proof. Let $\mu^{0}(s) \in C^{0}(\Gamma)$ be a solution of the homogeneous equation $\left(12^{\prime}\right)$. The kernel of the integral term in $\left(12^{\prime}\right)$ is continuous in $s, \sigma$ on $\Gamma$. It is shown in Appendix 3 (see Theorem A3) that the integral term in $\left(12^{\prime}\right)$ belongs to $C^{0,1 / 3}(\Gamma)$ in $s$; therefore $\mu^{0}(s) \in C^{0,1 / 3}(\Gamma)$. Now we consider the function $u\left[\mu^{0}\right](x)$ introduced in (6). This function satisfies the following homogeneous Dirichlet problem for the Laplace equation:

$$
\Delta u=0 \text { in } \mathcal{D},\left.\quad u\right|_{\Gamma}=0, \quad u(x) \in C^{0}(\overline{\mathcal{D}}) \cap C^{2}(\mathcal{D}) .
$$

Indeed, substituting $u\left[\mu^{0}\right](x)$ in the boundary condition, we get the identity $\left(12^{\prime}\right)$. According to the uniqueness theorem for the Dirichlet problem (A2.1) (it follows from the maximum principle for harmonic functions), we obtain

$$
u\left[\mu^{0}\right](x) \equiv 0, \quad x \in \overline{\mathcal{D}} .
$$

We consider the function

$$
\begin{aligned}
u^{*}\left[\mu^{0}\right](x)=-\frac{1}{2 \pi}[ & -\int_{\Gamma} \mu^{0}(\sigma) \frac{\partial}{\partial \sigma} \ln |x-y(\sigma)| d \sigma \\
& \left.+\sum_{n=2}^{N} \int_{\Gamma_{n}} \mu^{0}(\sigma) d \sigma \psi\left(x, Y_{n}\right)\right],
\end{aligned}
$$


where $\psi(x, y)$ is the polar angle of the polar system of coordinates with the center in the point $y$. If $N=1$, then the sum in (A2.3) is absent. The function $u^{*}\left[\mu^{0}\right](x)$ is connected with $u\left[\mu^{0}\right](x)$ by the Cauchy-Riemann relations $\partial_{x_{1}} u=\partial_{x_{2}} u^{*}, \partial_{x_{2}} u=-\partial_{x_{1}} u^{*}$, and due to $(\mathrm{A} 2.2)$ :

$$
u^{*}\left[\mu^{0}\right](x) \equiv C=\text { const }, \quad x \in \mathcal{D} .
$$

It is clear from (A2.3) that $u^{*}\left[\mu^{0}\right](x)$ is a multi-valued function, because $\psi\left(x, Y_{n}\right)$ are multi-valued functions $(n=2, \ldots, N)$. Indeed, when passing around the point $Y_{n}$ the value of the function $\psi\left(x, Y_{n}\right)$ changes for $2 \pi$. Evidently, $u^{*}\left[\mu^{0}\right](x)$ can be constant in $\mathcal{D}$ only if $u^{*}\left[\mu^{0}\right](x)$ is single-valued. In order for $u^{*}\left[\mu^{0}\right](x)$ to be single-valued, the following $N-1$ conditions must hold:

$$
\int_{\Gamma_{n}} \mu^{0}(\sigma) d \sigma=0, \quad n=2, \ldots, N .
$$

Under these conditions $u^{*}\left[\mu^{0}\right](x)$ takes the form of the modified single-layer potential [7. $\S 12]$

$$
u^{*}\left[\mu^{0}\right](x)=\frac{1}{2 \pi} \int_{\Gamma} \mu^{0}(\sigma) \frac{\partial}{\partial \sigma} \ln |x-y(\sigma)| d \sigma,
$$

and $u\left[\mu^{0}\right](x)$ transforms to the ordinary double-layer potential

$$
\begin{gathered}
u\left[\mu^{0}\right](x)=-\frac{1}{2 \pi} \int_{\Gamma} \mu^{0}(\sigma) \frac{\partial}{\partial \mathbf{n}_{y}} \ln |x-y(\sigma)| d \sigma \\
\in C^{0}(\overline{\mathcal{D}}) \cap C^{0}\left(\overline{R^{2} \backslash \mathcal{D}}\right) \cap C^{2}\left(R^{2} \backslash \Gamma\right) .
\end{gathered}
$$

The potentials (A2.5) and (A2.6) are connected by the Cauchy-Riemann relations in $R^{2} \backslash \Gamma$. Because of $\mu^{0}(s) \in C^{0,1 / 3}(\Gamma)$, the potential (A2.5) is a harmonic function, which belongs to $C^{0}\left(R^{2}\right) \cap C^{2}\left(R^{2} \backslash \Gamma\right)$ (see [7, $\left.\S 12, \S 16, \S 64\right]$ for details). Note that (A2.5) is continuous when passing through $\Gamma$ and is represented on $\Gamma$ by a singular integral (for this we stressed that $\mu^{0}(s)$ is a Hölder continuous function).

As stated above, $u^{*}\left[\mu^{0}\right](x) \equiv C$ in $\overline{\mathcal{D}}$.

We consider the interior domain $\mathcal{D}_{n}$ bounded by $\Gamma_{n}(n=2, \ldots, N)$. In this domain the potential (A2.5) satisfies the following Dirichlet problem:

$$
\Delta u^{*}=0 \quad \text { in } \quad \mathcal{D}_{n},\left.\quad u^{*}\right|_{\Gamma_{n}}=C, \quad u^{*}(x) \in C^{0}\left(\overline{\mathcal{D}_{n}}\right) \cap C^{2}\left(\mathcal{D}_{n}\right),
$$

which has the unique solution

$$
u^{*}\left[\mu^{0}\right](x) \equiv C, \quad x \in \overline{\mathcal{D}_{n}} \quad(n=2, \ldots, N) .
$$

It follows from the Cauchy-Riemann relations and the smoothness of the double-layer potential that

$$
u\left[\mu^{0}\right](x) \equiv c_{n}, \quad x \in \overline{\mathcal{D}_{n}}, \quad n=2, \ldots, N,
$$

where $c_{2}, \ldots, c_{N}$ are constants. Using (A2.2) and the jump relation for the double-layer potential $u\left[\mu^{0}\right](x)$ on $\Gamma$, we get

$$
\left.\mu^{0}(s)\right|_{\Gamma_{n}} \equiv-c_{n}, \quad n=2, \ldots, N .
$$

According to (A2.4), $c_{n}=0$ for $n=2, \ldots, N$. Therefore

$$
\left.\mu^{0}(s)\right|_{\Gamma_{n}} \equiv 0, \quad n=2, \ldots, N .
$$


We consider the exterior domain $\mathcal{D}_{1}$ bounded by $\Gamma_{1}$. In this domain the potential (A2.5) satisfies the following exterior Dirichlet problem:

$$
\begin{gathered}
\Delta u^{*}=0 \text { in } \mathcal{D}_{1},\left.\quad u^{*}\right|_{\Gamma_{1}}=C, \quad\left|u^{*}(x)\right|<\text { const in } \mathcal{D}_{1}, \\
u^{*}(x) \in C^{0}\left(\overline{\mathcal{D}_{1}}\right) \cap C^{2}\left(\mathcal{D}_{1}\right),
\end{gathered}
$$

which has the unique solution $u^{*}\left[\mu^{0}\right](x) \equiv C$. It follows from the Cauchy-Riemann relations and the smoothness of the double-layer potential that

$$
u\left[\mu^{0}\right](x) \equiv c_{1}, \quad x \in \overline{\mathcal{D}_{1}},
$$

where $c_{1}$ is a constant. Because of (A2.6) tending to zero at infinity, we have $c_{1}=0$ and $u\left[\mu^{0}\right](x) \equiv 0$ in $\overline{\mathcal{D}_{1}}$. Using (A2.2) and the theorem on the jump for the double-layer potential [9], we obtain $\left.\mu^{0}(s)\right|_{\Gamma_{1}} \equiv 0$. Taking into account (A2.7), we have

$$
\mu^{0}(s) \equiv 0 \quad \text { on } \Gamma \text {. }
$$

Hence, the homogeneous equation $\left(12^{\prime}\right)$ has only the trivial solution. Theorem A2 is proved.

Because $\left(12^{\prime}\right)$ is a Fredholm equation of the second kind and of index zero, the following corollary holds.

Corollary. If $\Gamma \in C^{2,0}$, then the inhomogeneous Fredholm equation $\left(12^{\prime}\right)$ is uniquely solvable in $C^{0}(\Gamma)$ for any right-hand side from $C^{0}(\Gamma)$.

Appendix 3. Let us study smoothness of the direct value of the double layer potential on the closed curve.

Let $\gamma$ be a closed curve of class $C^{2,0}$, and let $\mu(x)$ be a function, which is continuous in $x$ on $\gamma$. Consider the direct value of the double layer potential on $\gamma$ :

$$
I_{1}(x)=-\frac{1}{2 \pi} \int_{\gamma} \mu(y) \frac{\partial \ln |x-y|}{\partial \mathbf{n}_{y}} d l_{y}, \quad x \in \gamma,
$$

where the curvilinear integral of the first kind is taken over $\gamma$.

Lemma. Let $\gamma$ be a simple closed curve of class $C^{2,0}$, and let $\gamma_{1}$ be an arbitrary open arc, which is a part of $\gamma$ (i.e. $\left.\gamma_{1} \subset \gamma, \gamma_{1} \not \equiv \gamma\right)$. Let $\mu(x)$ be a function, which is continuous in $x$ on $\gamma$. Then $I_{1}(x)$ belongs to $C^{0,1 / 3}\left(\gamma_{1}\right)$ in $x$.

Proof. We introduce parametrization of $\gamma$ by the arc length $s$,

$$
\gamma=\{x: x=x(s), s \in[a, b]\},
$$

so that the point $x(a)=x(b) \in \gamma$ is situated outside $\gamma_{1}$, i.e. $x(a)=x(b) \notin \gamma_{1}, \gamma_{1}=\{x$ : $x=x(s), s \in[c, d]\}, a<c<d<b$. Then $\mu(x(s)) \in C^{0}[a, b]$ and $\mu(x(a))=\mu(x(b))$. Set

$$
\mathcal{I}_{1}(s)=I_{1}(x(s))=-\frac{1}{2 \pi} \int_{a}^{b} \mu(y(\sigma)) \frac{\partial \ln |x(s)-y(\sigma)|}{\partial \mathbf{n}_{y}} d \sigma, \quad x(s) \in \gamma .
$$


Let us prove that $\mathcal{I}_{1}(s) \in C^{0,1 / 3}[c, d]$. Taking into account that $\mathbf{n}_{y}=\left(y_{2}^{\prime}(\sigma),-y_{1}^{\prime}(\sigma)\right)$, we find

$$
\begin{gathered}
\frac{\partial \ln |x(s)-y(\sigma)|}{\partial \mathbf{n}_{y}}=\frac{T(s, \sigma)}{g(s, \sigma)}, \quad g(s, \sigma)=\frac{|x(s)-y(\sigma)|^{2}}{(s-\sigma)^{2}}, \\
T(s, \sigma)=\frac{\left[x_{2}(s)-y_{2}(\sigma)\right] y_{1}^{\prime}(\sigma)-\left[x_{1}(s)-y_{1}(\sigma)\right] y_{2}^{\prime}(\sigma)}{(s-\sigma)^{2}} .
\end{gathered}
$$

Note that $y(\sigma)$ is a point on $\gamma$, corresponding to $s=\sigma$. So, we may put $x(\sigma)=y(\sigma)$. For $j=1,2$ we have [18, $\S 3$ ]

$$
x_{j}(s)-x_{j}(\sigma)=(s-\sigma) Z_{j}^{1}(s, \sigma),
$$

where

$$
Z_{j}^{1}(s, \sigma)=\int_{0}^{1} x_{j}^{\prime}[\sigma+\xi(s-\sigma)] d \xi \in C^{1}([a, b] \times[a, b]) .
$$

Note that the function

$$
g(s, \sigma)=\frac{|x(s)-x(\sigma)|^{2}}{(s-\sigma)^{2}}=\left\{\left[Z_{1}^{1}(s, \sigma)\right]^{2}+\left[Z_{2}^{1}(s, \sigma)\right]^{2}\right\} \in C^{1}([a, b] \times[a, b])
$$

does not equal zero if $s \in[c, d], \sigma \in[a, b]$. In addition, $g(s, s)=1$. Therefore $\frac{1}{g(s, \sigma)} \in$ $C^{1}([c, d] \times[a, b])$. Similarly,

$$
\begin{aligned}
T(s, \sigma) & =\frac{\left[x_{2}(s)-x_{2}(\sigma)\right] x_{1}^{\prime}(\sigma)-\left[x_{1}(s)-x_{1}(\sigma)\right] x_{2}^{\prime}(\sigma)}{(s-\sigma)^{2}} \\
& =\frac{\mathcal{K}(s, \sigma)}{s-\sigma},
\end{aligned}
$$

where

$$
\mathcal{K}(s, \sigma)=\left[Z_{2}^{1}(\sigma, s) x_{1}^{\prime}(\sigma)-Z_{1}^{1}(\sigma, s) x_{2}^{\prime}(\sigma)\right] \in C^{1}([a, b] \times[a, b]) .
$$

From this representation it follows that $\mathcal{K}(s, s)=0$. According to [7, §5.7], the following representation holds:

$$
T(s, \sigma)=\frac{\mathcal{J}(s, \sigma)}{|s-\sigma|^{1 / 3}},
$$

where $\mathcal{J}(s, \sigma) \in C^{0,1 / 3}([a, b] \times[a, b])$. Hence

$$
\frac{\partial \ln |x(s)-y(\sigma)|}{\partial \mathbf{n}_{y}}=\frac{T(s, \sigma)}{g(s, \sigma)}=\frac{J(s, \sigma)}{|s-\sigma|^{1 / 3}},
$$

where $J(s, \sigma)=\frac{\mathcal{J}(s, \sigma)}{g(s, \sigma)} \in C^{0,1 / 3}([c, d] \times[a, b])$. Consequently,

$$
\begin{aligned}
\mathcal{I}_{1}(s) & =-\frac{1}{2 \pi} \int_{a}^{b} \mu(y(\sigma)) \frac{\partial \ln |x(s)-y(\sigma)|}{\partial \mathbf{n}_{y}} d \sigma \\
& =-\frac{1}{2 \pi} \int_{a}^{b} \mu(y(\sigma)) \frac{J(s, \sigma)}{|s-\sigma|^{1 / 3}} d \sigma .
\end{aligned}
$$


Let $s_{1}, s_{2} \in[c, d]$. Consider

$$
\begin{gathered}
\left|\mathcal{I}_{1}\left(s_{2}\right)-\mathcal{I}_{1}\left(s_{1}\right)\right| \leq\|\mu\|_{C^{0}(\gamma)} \frac{1}{2 \pi} \int_{a}^{b}\left|\frac{J\left(s_{2}, \sigma\right)}{\left|s_{2}-\sigma\right|^{1 / 3}}-\frac{J\left(s_{1}, \sigma\right)}{\left|s_{1}-\sigma\right|^{1 / 3}}\right| d \sigma \\
\leq\|\mu\|_{C^{0}(\gamma)} \frac{1}{2 \pi} \int_{a}^{b} \mid \frac{\left(J\left(s_{2}, \sigma\right)-J\left(s_{1}, \sigma\right)\right)\left|s_{1}-\sigma\right|^{1 / 3}}{\left|s_{2}-\sigma\right|^{1 / 3}\left|s_{1}-\sigma\right|^{1 / 3}} \\
-\frac{J\left(s_{1}, \sigma\right)\left(\left|s_{2}-\sigma\right|^{1 / 3}-\left|s_{1}-\sigma\right|^{1 / 3}\right)}{\left|s_{2}-\sigma\right|^{1 / 3}\left|s_{1}-\sigma\right|^{1 / 3}} \mid d \sigma \\
\leq\|\mu\|_{C^{0}(\gamma)} \frac{1}{2 \pi}\left(\int_{a}^{b} \frac{\left|J\left(s_{2}, \sigma\right)-J\left(s_{1}, \sigma\right)\right| \cdot\left|s_{1}-\sigma\right|^{1 / 3}}{\left|s_{2}-\sigma\right|^{1 / 3}\left|s_{1}-\sigma\right|^{1 / 3}} d \sigma\right. \\
\left.\quad+\int_{a}^{b} \frac{\left|J\left(s_{1}, \sigma\right)\right| \cdot|| s_{2}-\left.\sigma\right|^{1 / 3}-\left|s_{1}-\sigma\right|^{1 / 3} \mid}{\left|s_{2}-\sigma\right|^{1 / 3}\left|s_{1}-\sigma\right|^{1 / 3}} d \sigma\right) \\
\leq c_{0}\|\mu\|_{C^{0}(\gamma)}\left|s_{2}-s_{1}\right|^{1 / 3} \int_{a}^{b} \frac{1}{\left|s_{2}-\sigma\right|^{1 / 3}\left|s_{1}-\sigma\right|^{1 / 3}} d \sigma
\end{gathered}
$$

where $c_{0}$ is a constant. The properties of Hölder functions [7, $\left.\S 3.1, \S 5\right]$ are used when deriving the latter inequality. Using the Cauchy-Bunyakovskii inequality, we obtain

$$
\begin{gathered}
\int_{a}^{b} \frac{1}{\left|s_{2}-\sigma\right|^{1 / 3}\left|s_{1}-\sigma\right|^{1 / 3}} d \sigma \\
\leq\left(\int_{a}^{b} \frac{d \sigma}{\left|s_{2}-\sigma\right|^{2 / 3}}\right)^{1 / 2}\left(\int_{a}^{b} \frac{d \sigma}{\left|s_{1}-\sigma\right|^{2 / 3}}\right)^{1 / 2} \leq \mathrm{const}
\end{gathered}
$$

for any $s_{1}, s_{2} \in[c, d]$. Hence

$$
\left|\mathcal{I}_{1}\left(s_{2}\right)-\mathcal{I}_{1}\left(s_{1}\right)\right| \leq c_{2}\|\mu\|_{C^{0}(\gamma)}\left|s_{2}-s_{1}\right|^{1 / 3}, \quad s_{1}, s_{2} \in[c, d],
$$

where $c_{2}=c_{0} \cdot$ const is a constant. Therefore $\mathcal{I}_{1}(s) \in C^{0,1 / 3}[c, d]$ for any density $\mu(x) \in$ $C^{0}(\gamma)$.

Moreover, for any $x^{1}, x^{2} \in \gamma_{1}$, where $x^{1}=x\left(s_{1}\right), x^{2}=x\left(s_{2}\right)$, and $s_{1}, s_{2} \in[c, d]$, we obtain

$$
\begin{gathered}
\left|I_{1}\left(x^{2}\right)-I_{1}\left(x^{1}\right)\right|=\left|I_{1}\left(x\left(s_{2}\right)\right)-I_{1}\left(x\left(s_{1}\right)\right)\right|=\left|\mathcal{I}_{1}\left(s_{2}\right)-\mathcal{I}_{1}\left(s_{1}\right)\right| \\
\leq c_{2}\|\mu\|_{C^{0}(\gamma)}\left|s_{2}-s_{1}\right|^{1 / 3} \leq c_{2}\|\mu\|_{C^{0}(\gamma)} \frac{\left|s_{2}-s_{1}\right|^{1 / 3}}{\left|x\left(s_{2}\right)-x\left(s_{1}\right)\right|^{1 / 3}}\left|x\left(s_{2}\right)-x\left(s_{1}\right)\right|^{1 / 3} \\
\leq c_{2} \cdot c_{1}^{1 / 6}\|\mu\|_{C^{0}(\gamma)}\left|x\left(s_{2}\right)-x\left(s_{1}\right)\right|^{1 / 3}=c_{2} \cdot c_{1}^{1 / 6}\|\mu\|_{C^{0}(\gamma)}\left|x^{2}-x^{1}\right|^{1 / 3} .
\end{gathered}
$$

Here we used the inequality

$$
\frac{1}{g\left(s_{2}, s_{1}\right)}=\frac{\left|s_{2}-s_{1}\right|^{2}}{\left|x\left(s_{2}\right)-x\left(s_{1}\right)\right|^{2}} \leq c_{1}
$$

for all $s_{1}, s_{2} \in[c, d]$, where $c_{1}$ is a constant. This inequality is true since $\frac{1}{g(s, \sigma)} \in$ $C^{1}([c, d] \times[a, b])$, as was shown above. It follows from (A3.1) that $I_{1}(x) \in C^{0,1 / 3}\left(\gamma_{1}\right)$ in the variable $x$. The lemma is proved. 
Theorem A3. Let $\gamma$ be a simple closed curve of class $C^{2,0}$, parametrized by the arc length $s: \gamma=\{x: x=x(s), s \in[a, b], x(a)=x(b)\}$, and

$$
\mu(x(s)) \in C^{0}(\gamma)=\left\{\mu(x(s)) \in C^{0}[a, b], \mu(x(a))=\mu(x(b))\right\} .
$$

Let

$$
\mathcal{I}_{1}(s)=I_{1}(x(s))=-\frac{1}{2 \pi} \int_{a}^{b} \mu(y(\sigma)) \frac{\partial \ln |x(s)-y(\sigma)|}{\partial \mathbf{n}_{y}} d \sigma
$$

be the direct value of the double layer potential on $\gamma$. Then

$$
\begin{gathered}
\mathcal{I}_{1}(s) \in C^{0,1 / 3}(\gamma) \\
=\left\{\mathcal{I}_{1}(s) \in C^{0,1 / 3}[a, b], \quad \mathcal{I}_{1}(a)=\mathcal{I}_{1}(b)\right\} .
\end{gathered}
$$

Proof. Since $\gamma_{1}$ in the lemma is an arbitrary nonclosed arc lying on $\gamma$, we subdivide the curve $\gamma$ into two parts and observe that $I_{1}(x)$ is a Hölder continuous function in $x$ with exponent $1 / 3$ on each part. Note that $I_{1}(x)$ is continuous on $\gamma$ in $x$. Repeating the arguments from [7, §5.1], we observe that $I_{1}(x) \in C^{0,1 / 3}(\gamma)$ in $x$. Hence, for all $s_{1}, s_{2} \in[a, b]$ and for some constant $c_{3}$ we have

$$
\begin{gathered}
\left|\mathcal{I}_{1}\left(s_{2}\right)-\mathcal{I}_{1}\left(s_{1}\right)\right|=\left|I_{1}\left(x\left(s_{2}\right)\right)-I_{1}\left(x\left(s_{1}\right)\right)\right| \leq c_{3}\left|x\left(s_{2}\right)-x\left(s_{1}\right)\right|^{1 / 3} \\
\leq c_{3} \frac{\left|x\left(s_{2}\right)-x\left(s_{1}\right)\right|^{1 / 3}}{\left|s_{2}-s_{1}\right|^{1 / 3}}\left|s_{2}-s_{1}\right|^{1 / 3} \leq c_{3} c_{4}^{1 / 6}\left|s_{2}-s_{1}\right|^{1 / 3},
\end{gathered}
$$

where $c_{4}$ is a constant. So, $\mathcal{I}_{1}(s) \in C^{0,1 / 3}[a, b]$ in $s$. When deriving the latter inequality we used the fact that

$$
\frac{\left|x\left(s_{2}\right)-x\left(s_{1}\right)\right|^{2}}{\left|s_{2}-s_{1}\right|^{2}}=g\left(s_{2}, s_{1}\right) \leq c_{4}=\text { const }
$$

for all $s_{1}, s_{2} \in[a, b]$. Indeed, it was shown in the proof of the lemma that $g(s, \sigma) \in$ $C^{1}([a, b] \times[a, b])$, whence it follows that the function $g(s, \sigma)$ is uniformly bounded in $s, \sigma$ on the set $[a, b] \times[a, b]$.

It is easy to verify that the kernel in the integral $I_{1}(x(s))$ depends on $x_{j}^{(m)}(s), j=1,2$; $m=0,1$. The values of these functions at $s=a$ and at $s=b$ are equal and the kernel is continuous in $x, y$ on $\gamma$. Therefore, $I_{1}(x(a))=I_{1}(x(b))$, so $\mathcal{I}_{1}(a)=\mathcal{I}_{1}(b)$. Thus, $\mathcal{I}(s) \in C^{0,1 / 3}(\gamma)$ in the variable $s$. That accomplishes the proof.

The author is grateful to Professor Heinrich Begehr for the opportunity to carry out research in Germany. The research has been partially supported by a DAAD scholarship and the RFBR grants 08-01-00082 and 09-01-00025.

\section{REFERENCES}

[1] Tovmasian N.E. On existence and uniqueness of a solution of the Dirichlet problem for the Laplace equation in classes of functions having singularities at the boundary of the domain. Siberian Mathematical Journal, 1961, v.2, No.2, pp.290-312 (in Russian). MR0124518 (23:A1830)

[2] Oganian V.A. The Dirichlet problem for elliptic systems of differential equations with discontinuous boundary conditions. Proceedings of Academi of Sciences of Armenian SSR, 1981, v.16, No.6, pp.465477 (in Russian). MR659978 (84a:35077)

[3] Krutitskii P.A. The Dirichlet problem for the 2-D Laplace equation in a multiply connected domain with cuts. Proc. Edinburgh Math. Soc., 2000, v.43, pp.325-341. MR.1763054 (2002h:31002) 
[4] Krutitskii P.A. On the harmonic Dirichlet problem on a two-dimensional domain with cuts. Doklady Akademii Nauk, 2007, v.415, No.1, pp.21-25. (in Russian). English translation in Doklady Mathematics, 2007, v.76, No.1, pp.497-501.

[5] Krutitskii P.A. On existence of a classical solution and non-existence of a weak solution to the Dirichlet problem in a planar domain with slits for Laplacian. Quarterly of Applied Mathematics, 2008, v.66, No. 1, pp.177-190. MR.2396656

[6] Krutitskii P.A. The mixed harmonic problem in a bounded cracked domain with Dirichlet condition on cracks. Journal of Differential Equations, 2004, v.198, pp.422-441. MR2039149 (2005b:35053)

[7] Muskhelishvili N.I. Singular integral equations. Nauka, Moscow, 1968 (in Russian). English translation: Noordhoff, Groningen, 1972. MR 0355494(50:7968)

[8] Tikhonov A.N., Samarskii A.A. Equations of mathematical physics. Pergamon Press, Oxford, 1963. MR0165209 (29:2498)

[9] Vladimirov V.S. Equations of mathematical physics. Nauka, Moscow, 1981 (in Russian). English translation: Mir Publishers, Moscow, 1984. MR764399 (86f:00030)

[10] Gilbarg D., Trudinger N.S. Elliptic partial differential equations of second order. Springer-Verlag, Berlin-New York, 1977. MR0473443 (57:13109)

[11] Krein S.G. (editor). Functional analysis. Nauka, Moscow, 1964 (in Russian). English translation: Wolters-Noordhoff Publishing, Groningen, 1972.

[12] Kantorovich L.V., Akilov G.P. Functional Analysis. Nauka, Moscow, 1984 (in Russian). English translation: Pergamon Press, Oxford, 1982. MR664597 (83h:46002)

[13] Trenogin V.A. Functional Analysis. Nauka, Moscow, 1980 (in Russian). French translation: Mir Publishers, Moscow, 1985. MR836334 (87g:46001)

[14] Bernardi C., Karageorghis A. L'équation de Laplace avec conditions aux limites discontinues: convergence d'une discrétisation par éléments spectraux. C. R. Acad. Sci. Paris Ser. I Math., 1997, v.324, No.10, pp.1161-1168. MR.1451941 (97m:65217)

[15] Iovanovich B. S. The difference method for solving the Dirichlet problem for the Laplace equation in the disc in case of discontinuous boundary conditions. Mat. Vesnik, 1981, v.5(18)(33), No.1, pp.69-79. MR681241(84d:65075)

[16] Berikelashvili G.K. A difference scheme of high-order accuracy for the solution of the Dirichlet problem for the Laplace equation with discontinuous boundary conditions. Soobsch. Akad. Nauk. Gruz. SSR, 1978, v.92, No.1, pp.29-32 (in Russian). MR540085(80h:65075)

[17] Arhipova E. Ju. A difference Dirichlet problem with discontinuous boundary conditions. Zh. Vychisl. Mat. i Mat. Fiz., 1976, v.16, No.1, pp.224-228 (in Russian). MR0418479 (54:6518)

[18] Krutitskii P.A. The Dirichlet problem for the Helmholtz equation outside slits in a plane. Comp. Maths. Math. Phys., 1994, v.34, No. 8/9, pp. 1073-1090. MR1300397 (95f:35046)

[19] Sobolev S. L. Some applications of functional analysis to mathematical physics. Nauka, Moscow, 1988 (in Russian). MR986735 (90m:46059) 